\title{
Increased antiparkinson efficacy of the combined administration of VEGF- and GDNF-loaded nanospheres in a partial lesion model of Parkinson's disease
}

This article was published in the following Dove Press journal:

International Journal of Nanomedicine

27 May 2014

Number of times this article has been viewed

\author{
Enara Herrán ${ }^{1,2}$ \\ Catalina Requejo ${ }^{3}$ \\ Jose Angel Ruiz-Ortega ${ }^{4}$ \\ Asier Aristieta ${ }^{4}$ \\ Manoli Igartua ${ }^{1,2}$ \\ Harkaitz Bengoetxea ${ }^{3}$ \\ Luisa Ugedo ${ }^{4}$ \\ Jose Luis Pedraz ${ }^{1,2}$ \\ Jose Vicente Lafuente ${ }^{3}$ \\ Rosa Maria Hernández ${ }^{1,2}$ \\ 'NanoBioCel Group, Laboratory \\ of Pharmaceutics, University of \\ the Basque Country (UPV/EHU), \\ School of Pharmacy, Vitoria, Spain; \\ ${ }^{2}$ Biomedical Research Networking \\ Center in Bioengineering, Biomaterials \\ and Nanomedicine (CIBER-BBN) \\ Vitoria, Spain; ${ }^{3}$ LaNCE, Department \\ of Neurosciences, University of the \\ Basque Country (UPV/EHU), Leioa, \\ Spain; ${ }^{4}$ Department of Pharmacology, \\ University of the Basque Country \\ (UPV/EHU), Leioa, Spain
}

\begin{abstract}
Current research efforts are focused on the application of growth factors, such as glial cell line-derived neurotrophic factor (GDNF) and vascular endothelial growth factor (VEGF), as neuroregenerative approaches that will prevent the neurodegenerative process in Parkinson's disease. Continuing a previous work published by our research group, and with the aim to overcome different limitations related to growth factor administration, VEGF and GDNF were encapsulated in poly(lactic-co-glycolic acid) nanospheres (NS). This strategy facilitates the combined administration of the VEGF and GDNF into the brain of 6-hydroxydopamine (6-OHDA) partially lesioned rats, resulting in a continuous and simultaneous drug release. The NS particle size was about $200 \mathrm{~nm}$ and the simultaneous addition of VEGF NS and GDNF NS resulted in significant protection of the PC-12 cell line against 6-OHDA in vitro. Once the poly(lactic-co-glycolic acid) NS were implanted into the striatum of 6-OHDA partially lesioned rats, the amphetamine rotation behavior test was carried out over 10 weeks, in order to check for in vivo efficacy. The results showed that VEGF NS and GDNF NS significantly decreased the number of amphetamine-induced rotations at the end of the study. In addition, tyrosine hydroxylase immunohistochemical analysis in the striatum and the external substantia nigra confirmed a significant enhancement of neurons in the VEGF NS and GDNF NS treatment group. The synergistic effect of VEGF NS and GDNF NS allows for a reduction of the dose by half, and may be a valuable neurogenerative/neuroreparative approach for treating Parkinson's disease.
\end{abstract}

Keywords: nanoparticles, PLGA, 6-OHDA, neuroregeneration, neurotrophic factors, tyrosine hydroxylase

\section{Introduction}

In recent years, there has been increasing interest in the struggle against neurodegenerative diseases such as Parkinson's disease (PD). PD is characterized by selective degeneration of the nigrostriatal pathway and a concomitant reduction in the striatal concentration of dopamine, giving rise to motor function impairments such as rigidity, rest tremors, bradykinesia, and postural abnormalities. ${ }^{1,2}$

Although PD is a major priority for health care systems, current dopamimetic therapies, such as L-dopa, focus on modifying motor symptoms without treating the neurodegenerative process and without providing neuroprotection to the surviving dopaminergic neurons. ${ }^{3}$ Thus, current research efforts are focused on halting neurodegeneration using promising alternatives such as antioxidants, antiapoptotic agents, cell-based therapies, and neuroprotective agents. ${ }^{4}$

An interesting and promising approach is the use of growth factors (GFs), and specifically the subfamily of the neurotrophic factors (NTFs), which are potentially

\footnotetext{
Correspondence: Rosa Maria Hernández NanoBioCel Group, Laboratory of Pharmaceutics, University of the Basque Country (UPV/EHU), School of Pharmacy, Paseo de la Universidad 7, 01006,Vitoria, Spain

Tel +349450I3095

Fax +34945013040

Email rosa.hernandez@ehu.es
} 
major players in therapeutic interventions for neurodegenerative disorders such as PD. ${ }^{5}$ NTFs represent one of the most important challenges in the treatment of neurodegenerative diseases due to their roles in the survival and phenotypic differentiation of developing neurons, as well as maintenance and protection of mature and injured neurons. ${ }^{4,6}$

According to the scientific literature, and fueled by the positive results obtained in a previous study published by our group, ${ }^{7}$ we decided to continue with the application of NTFs in future works, and, in particular, with the promising combination of glial cell line-derived neurotrophic factor (GDNF) and vascular endothelial growth factor (VEGF). GDNF is a potent factor that is able to act in vitro and in vivo, promoting the survival and differentiation of dopaminergic neurons and protecting these cells from dopaminergic toxins. ${ }^{8-12}$ Several clinical trials have been conducted to analyze the potential of GDNF in PD patients; however, in all these studies, intracerebroventricularly or intraputaminally administered GDNF solution presented numerous negative side effects and no significant clinical improvements. ${ }^{13-15}$ VEGF has prosurvival effects in neuronal culture and was demonstrated to be protective against 6-hydroxydopamine (6-OHDA) in a PD rat model. In the present study, we used this potent angiogenic growth factor in combination with GDNF, in order to enhance the action of the latter..$^{16,17}$

Major problems for the clinical use of VEGF and GDNF is their rapid degradation rate, their short half-life in vivo, their difficulty in crossing the blood-brain barrier, and, consequently, the need for direct and continuous administration of the factors into the brain. ${ }^{18,19}$ One approach that has been examined by our group, ${ }^{7,20}$ to overcome these drawbacks is the encapsulation of VEGF and GDNF into biocompatible and biodegradable poly(lactic-co-glycolic acid) (PLGA) microspheres and nanospheres (NS). This strategy permits the intracranial administration of the formulations, allowing for a sustained drug release, with promising results obtained for PD and Alzheimer's disease recovery in preclinical studies. ${ }^{7,20}$ The intracranial administration of GDNF-loaded PLGA microspheres has also been demonstrated by other groups to be successful in improving behavioral deficits and reversing anatomical changes..$^{21,22}$

To appraise novel formulations in animal models of PD, various 6-OHDA lesioned rat models have been developed, in which the toxin was injected into different parts of the nigrostriatal pathway. In our previous work, ${ }^{7}$ we used a severely lesioned rat model of PD, whereas, in this new study, subregions in the caudoputamen complex were selected as a target for the lesion. This is a partial, more selective damage of the nigrostriatal dopaminergic pathway, which appears to be a particularly effective site in which to assess regeneration accompanied by recovery of motor behavior. ${ }^{23,24}$

Thus, in the present work, we studied the combined potential of low doses (half that of individual treatment) of VEGF-loaded PLGA NS and GDNF-loaded PLGA NS in a partially lesioned PD rat model, with the aim of studying their synergistic effects in neuronal recovery and protection.

\section{Materials and methods Preparation of NS}

Recombinant human VEGF (PeproTech, London, UK) and recombinant murine GDNF (Peprotech) enclosing PLGA (50:50; lactic/glycolic [\%]) (Resomer ${ }^{\circledR}$ RG 503; Boehringer Ingelheim, Ingelheim, Germany) NS were prepared as previously described. ${ }^{20}$

Briefly, 133 mg PLGA 50:50 (previously sterilized by gamma radiation) were dissolved in $3.33 \mathrm{~mL}$ dichloromethane and emulsified with $200 \mu \mathrm{L}$ of $0.15 \% \mathrm{w} / \mathrm{v}$ recombinant human VEGF or recombinant murine GDNF aqueous solution (containing 7\% [w/v] human serum albumin and $2.5 \%[\mathrm{w} / \mathrm{v}]$ poly-ethylene-glycol 400 ) by probe sonication for 30 seconds at $50 \mathrm{~W}\left(\right.$ Branson $^{\circledR}$ Sonifier $^{\circledR} 250$, Biogen, Derio, Spain). The first emulsion was poured into $5 \%(\mathrm{w} / \mathrm{v})$ polyvinyl alcohol solution and sonicated again for 1 minute, in order to obtain a double emulsion $\left(\mathrm{w}_{1} / \mathrm{o} / \mathrm{w}_{2}\right)$. This emulsion was poured into $2 \%(\mathrm{v} / \mathrm{v})$ isopropanol solution and stirred at room temperature for 2 hours to promote the removal of the organic solvent. The newly formed NS were separated by centrifugation at $20,000 \times g$, resuspended in $2.5 \%$ (w/w in respect to PLGA) trehalose aqueous solution and freeze-dried for 24 hours. The entire process of NS preparation was conducted under aseptic conditions. Empty NS (without VEGF or GDNF) were prepared using the same method described above.

\section{Characterization of NS}

The mean particle diameter, size distribution, and zeta potential were determined by the Malvern ${ }^{\circledR}$ Zetasizer Nano ZS (model Zen 3600; Malvern Instruments, Malvern, UK). Different surface characteristics of the NS were examined by scanning electron microscopy (JEOL ${ }^{\circledR}$ JSM-7000F; JEOL, Tokyo, Japan).

The encapsulation efficiencies of VEGF and GDNF were determined using a Human VEGF ELISA (enzymelinked immunosorbent assay) Development Kit (Peprotech) and the GDNF $\operatorname{Emax}^{\circledR}$ ImmunoAssay System (Promega Corporation, Fitchburg, WI, USA) after NS disruption with dimethyl sulfoxide. To evaluate the surface-associated protein, $3 \mathrm{mg}$ of NS were suspended in phosphatebuffered saline ([PBS] $\mathrm{pH} 7.4$ ) and maintained at $37^{\circ} \mathrm{C}$ 
for 30 minutes, under continuous orbital rotation. The resulting supernatant was collected and measured by the corresponding ELISA kit.

Finally, the release profile of VEGF and GDNF from PLGA NS was assessed. Three milligrams of NS was placed in an Eppendorf tube (containing $1 \mathrm{~mL}$ of PBS $20 \mathrm{mM}$ [pH 7.4]) at $37^{\circ} \mathrm{C} \pm 0.5^{\circ} \mathrm{C}$ and shaken with a rotator shaker at $25 \mathrm{rpm}$. At determined time intervals, the release medium was withdrawn by centrifugation $(20,000 \times g, 15$ minutes $)$ and replaced with $1 \mathrm{~mL}$ of fresh PBS. The amount of VEGF and GDNF in the supernatants was determined by VEGF ELISA and GDNF ELISA kits. The test was carried out in triplicate, representing the obtained results as the percentage of the total amount entrapped in the NS.

\section{Establishment of neurotoxic cell model with 6-OHDA}

This assay was conducted in the PC-12 Adh cell line (American Type Culture Collection [ATCC], Manassas, VA, USA), a specific cell lineage of dopaminergic neurons. PC-12 cells were sustained in specific medium (F-12K Gibco ${ }^{\circledR}$; Thermo Fisher Scientific, Waltham, MA, USA) supplemented with $2.5 \%$ fetal bovine serum, $15 \%$ horse serum, and $1 \%$ penicillin/ streptomycin in standard conditions. The culture plates were previously coated with attachment factor $\left(\mathrm{Gibco}^{\circledR}\right)$ in order to promote the attachment of seeded cells. After 24 hours, freshly prepared 6-OHDA (Sigma-Aldrich, St Louis, MO, USA), at different concentrations $(0.05 \mathrm{mM}, 0.1 \mathrm{mM}$, and $0.2 \mathrm{mM}$ ), was added into the cell culture. Cell viability was examined at 16 hours after the exposure to 6-OHDA, and CCK-8 (Cell Counting Kit-8 for quantization of viable cell number in proliferation and cytotoxicity assays; SigmaAldrich) and 4',6-diamidino-2-phenylindole (DAPI) immunostaining assays were conducted.

\section{Protective effect of VEGF and GDNF on PC- 12 cell cultures}

Once the 6-OHDA concentration was selected, VEGF and GDNF protection assays were also conducted in the PC-12 cell line. Cells were seeded in 96-well culture plates at a density of $150 \times 10^{3}$ cells/well, previously treated with an attachment factor, and maintained in standard conditions for 24 hours prior to experimentation. Then, culture medium was replaced, and cells were treated for 16 hours with 6-OHDA $(0.1 \mathrm{mM})$ and $5 \mathrm{ng} / \mathrm{mL}$ or $10 \mathrm{ng} / \mathrm{mL}$ of the factors released from the NS: 1) $0.1 \mathrm{mM}$ 6-OHDA; 2) $0.1 \mathrm{mM}$ 6-OHDA $+10 \mathrm{ng} / \mathrm{mL}$ of VEGF; 3) $0.1 \mathrm{mM}$ 6-OHDA +10 ng/mL of GDNF; 4) $0.1 \mathrm{mM}$ 6-OHDA +10 ng/mL VEGF +10 ng/mL GDNF; 5) $0.1 \mathrm{mM}$ 6-OHDA $+5 \mathrm{ng} / \mathrm{mL}$ VEGF $+5 \mathrm{ng} / \mathrm{mL}$ GDNF. The bioactivity of the samples was evaluated by measuring cell proliferation by adding $10 \mu \mathrm{L}$ of CCK-8 to each well.

\section{Animals and 6-OHDA lesion}

Thirty minutes before the surgery, male albino Sprague Dawley (150-175 g) rats were pretreated with desipramine (25 mg/kg, intraperitoneally [ip]; Sigma-Aldrich) and pargyline (50 mg/kg, ip; Sigma-Aldrich), to protect noradrenergic terminals and to inhibit monoamine oxidase activity, respectively. ${ }^{23}$

Then, rats were deeply anesthetized with isoflurane (1.5\%-2\%; Esteve Química, Barcelona, Spain) and placed on a Kopf stereotaxic instrument (David Kopf Instruments, Tujunga, CA, USA). 6-OHDA was unilaterally infused in the right striatum through three different injections. Seven point five micrograms of 6-OHDA were injected in each coordinate: anteroposterior $(\mathrm{AP})+1.3 \mathrm{~mm}$, mediolateral $(\mathrm{ML})+2.8 \mathrm{~mm}$, and dorsoventral (DV) $-4.5 \mathrm{~mm}$; AP $-0.2 \mathrm{~mm}, \mathrm{ML}+3.0 \mathrm{~mm}$, and DV $-5.0 \mathrm{~mm}$; AP $-0.6 \mathrm{~mm}$, $\mathrm{ML}+4.0 \mathrm{~mm}$, and $\mathrm{DV}-5.5 \mathrm{~mm}$, relative to bregma and dura with the tooth bar set at -2.4 . Taking into account the protocol, this lesion produces a partial degeneration (or parkinsonism). ${ }^{22}$

Two weeks after the 6-OHDA surgery, the rotational behavior was tested with amphetamine administration as a marker of dopaminergic degeneration. Only the rats that rotated more than three full turns/minute were included in this study.

\section{NS implantation surgery}

NS were implanted 3 weeks after the 6-OHDA injection. Rats were anesthetized with isoflurane (1.5\%-2\%; Esteve Química) and mounted on a Kopf stereotaxic instrument for NS implantation. Five different groups of animals were used (six to eight rats per group): 1) vehicle; 2) empty-NS; 3) VEGF NS (2.5 $\mu \mathrm{g}$ VEGF); 4) GDNF NS (2.5 $\mu \mathrm{g}$ GDNF); and 5) VEGF NS and GDNF NS (1.25 $\mu \mathrm{g}$ VEGF and $1.25 \mu \mathrm{g}$ GDNF). The NS were suspended in $15 \mu \mathrm{L}$ of PBS $(0.1 \% \mathrm{w} / \mathrm{v}$ carboxymethyl cellulose, $0.8 \% \mathrm{w} / \mathrm{v}$ Tween 80 [Sigma-Aldrich] and $0.8 \% \mathrm{w} / \mathrm{v}$ mannitol) before administration.

The NS were implanted in the right striatum in three different coordinates: $\mathrm{AP}+1.3 \mathrm{~mm}, \mathrm{ML}+2.8 \mathrm{~mm}$, and $\mathrm{DV}-4.5 \mathrm{~mm}$; $\mathrm{AP}-0.2 \mathrm{~mm}, \mathrm{ML}+3.0 \mathrm{~mm}$, and $\mathrm{DV}-5.0 \mathrm{~mm}$; AP $-0.6 \mathrm{~mm}$, $\mathrm{ML}+4.0 \mathrm{~mm}$, and DV $-5.5 \mathrm{~mm}$, relative to bregma and dura with the tooth bar set at -2.4 .

To carry out this in vivo assay, the rats were housed in usual conditions with a invariable temperature of $22^{\circ} \mathrm{C}$, a 12 -hour dark/light cycle and ad libitum access to water and food. All experimental methods were carried out in agreement 
with the Ethical Committee of Animal Welfare (CEBA) at the University of the Basque Country, Leioa, Spain (CEBA/154/2010//RUIZ ORTEGA).

\section{Behavioral study}

Amphetamine-induced turning behavior tests were developed $2,4,6,8$, and 10 weeks after the NS implantation, as previously described. ${ }^{24}$

Before each behavioral session, animals were weighed and amphetamine $(5 \mathrm{mg} / \mathrm{kg})$ was intraperitoneally administrated. After 15 minutes of latency, the full ipsilateral rotations were counted for 90 minutes with an automatized rotameter (multicounter LE3806; Harvard Apparatus, Holliston, MA, USA). Data were expressed as the number of full turns per minute.

Fourteen weeks after NS implantation, apomorphineinduced rotational behavior was tested. The apomorphine (a dopaminergic agonist) was used as a dopamine (DA) degeneration indicator because it induces contralateral turning behavior after dopaminergic denervation. Thus, before each behavioral session, animals were weighed and apomorphine $(0.5 \mathrm{mg} / \mathrm{kg})$ was subcutaneously administrated. After 5 minutes of latency, the full contralateral rotations were measured for 50 minutes with an automatized rotameter (Harvard Apparatus multicounter LE3806). Data were expressed as the number of full turns per 5 minutes.

Figure 1 shows a schematic illustration of the experimental design for the treatments in 6-OHDA lesioned rats.

\section{Tyrosine hydroxylase $(\mathrm{TH})$ immunohistochemistry}

Animals underwent transcardial perfusion with saline solution and 4\% paraformaldehyde in 0.1 M PBS, pH 7.4. Brains were removed and postfixed for 48 hours in the same fixative solution and then cryopreserved in $30 \%$ sucrose diluted in 0.1 M PBS. Three days after, brains were sectioned on a freezing microtome ( $52 \mu \mathrm{m}$ thick) and coronal slices collected in PBS containing $0.6 \%$ sodium azide. TH immunochemistry assay was performed in free-floating slices and used to evaluate dopamine loss in basal ganglia and substantia nigra (SN). For TH immunostaining, we neutralized endogenous peroxidases with a solution of 3\% $\mathrm{H}_{2} \mathrm{O}_{2}, 10 \%$ methanol in potassium PBS (KPBS) $(0.02 \mathrm{M}$, $\mathrm{pH}$ 7.1) for 30 minutes at room temperature. Cerebral slices were preincubated with $5 \%$ normal goat serum (NGS) and $1 \%$ Triton $^{\mathrm{TM}} \mathrm{X}-100$ (Sigma-Aldrich) in KPBS (KPBS/T) for 1 hour and then incubated overnight with rabbit polyclonal anti-TH (1:1000) in 5\% NGS KPBS/T at $4{ }^{\circ} \mathrm{C}$. After rinsing twice with KPBS and once with $2.5 \%$ NGS KPBS/T, slices were incubated for 2 hours with a secondary biotinylated goat anti-rabbit immunoglobulin G (1:200 in KPBS/T containing 2.5\% NGS). All slices were processed with avidin-biotin-peroxidase complex (Elite ABC kit; Vector Laboratories, Inc., Burlingame, CA, USA) for 1 hour with 3,3'-diaminobenzidine (DAB) as the chromogen. Finally, slices were mounted, dehydrated, and coverslipped with permanent mounting DPX medium (Sigma-Aldrich).

\section{Integrated optical density (IOD)}

The optical density of basal ganglia was evaluated using a computerized image analysis system (Mercator Image Analysis system; Explora Nova, La Rochelle, France). Digitalized images from coronal sections were taken with a 1,200 dpi resolution digital scanner (Epson, Suwa, Japan). The IOD reading was corrected for background staining (subtracting the values of a region outside of the tissue). For each animal, the IOD was estimated at two medial-caudal levels along the basal ganglia according to Paxinos and Watson, ${ }^{25}$ placing a square of $900 \mu \mathrm{m}^{2}$ on the most degenerated lateral region: 1) medial striatum (bregma $-0.24 \mathrm{~mm}$ ); 2) caudal striatum

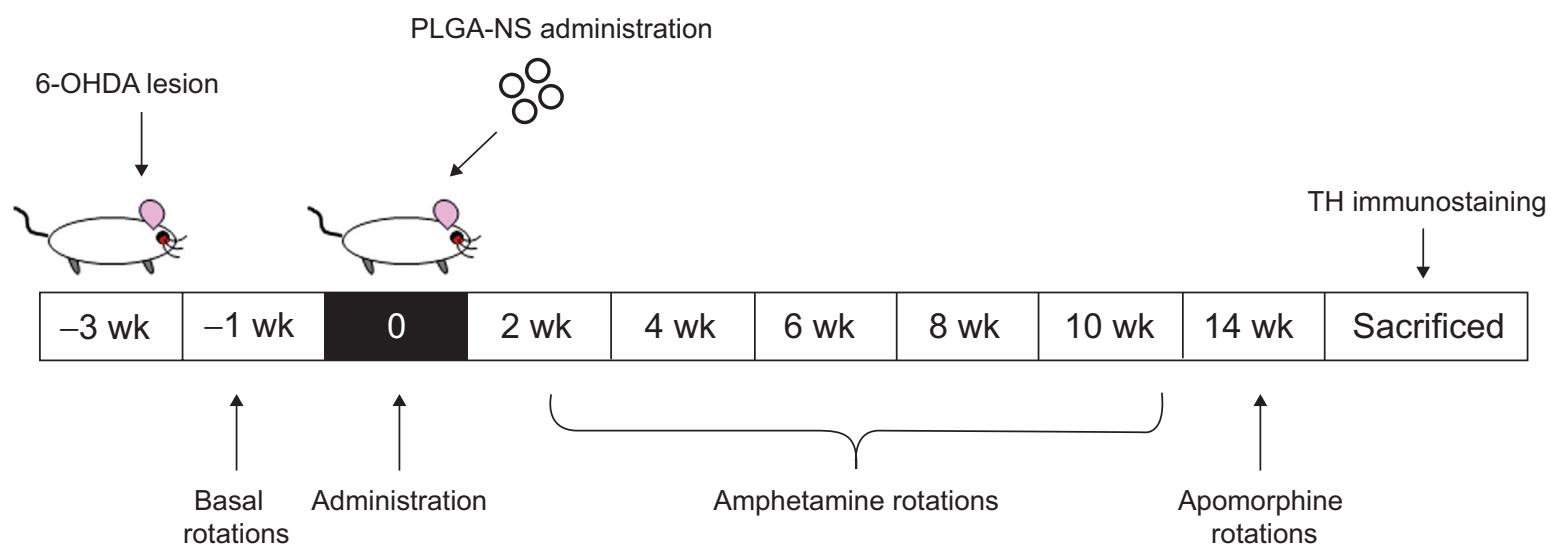

Figure I Experimental design for the NS treatments in 6-OHDA lesioned rats.

Abbreviations: 6-OHDA, 6-hydroxydopamine; NS, nanosphere; PLGA, poly(lactic-co-glycolic acid); TH, tyrosine hydroxylase; wk, weeks. 
(bregma $-0.60 \mathrm{~mm}$ ). Optical density values are given as percentage of ipsilateral striatum versus the contralateral non-lesioned striatum, which was considered as $100 \%$.

\section{Number of neurons}

Immunoreactive neurons were measured using a stereological tool (optical dissector) provided by the computerized image analysis system (Mercator Image Analysis system; Explora Nova, La Rochelle, France). Probes of $50 \times 50 \mu \mathrm{m}$ separated by $100 \mu \mathrm{m}$ were launched into the previously delimited region belonging to the lateral region of the $\mathrm{SN}$. This region is topologically related to the dorsolateral caudoputamen complex, where the 6-OHDA lesion was produced. $^{21,26}$

Immunopositive neurons inside the probe or crossing on the right side of the $\mathrm{X}-\mathrm{Y}$ axis were counted. A minimum of three histological sections per animal and five animals from each experimental group were analyzed. Density of neurons per slice and per animal were calculated.

\section{Statistical analysis}

All statistical analysis was performed with GraphPad Prism (v 5; GraphPad Software, Inc., La Jolla, CA, USA) and SPSS Statistics (v 20; IBM Corporation, Armonk, NY, USA). Prior to analysis, the Shapiro-Wilk test was used to assess normal distribution of samples, and Levene's test was used to determine the homogeneity of variance. One-way analysis of variance (ANOVA) with Tamhane's post hoc test was used to explore differences between groups. In the behavioral studies, we used Student's $t$-test to compare intergroup differences. Significance was declared when $P<0.05$.

\section{Results}

\section{Characterization of NS}

NS presented particle sizes of around $235.6 \pm 0.111 \mathrm{~nm}$ for VEGF NS, $221.1 \pm 0.065 \mathrm{~nm}$ for GDNF NS, and $267.00 \pm 0.111 \mathrm{~nm}$ for empty NS. The zeta potential was similar for all the formulations, around $-25 \mathrm{mV}$. When visualized by scanning electron microscopy, the NS showed a spherical shape without irregularities (Figure 2). The encapsulation efficiency (EE) was 44\% for VEGF NS and was higher for GDNF NS, at 74\% (Figure 2A and Table 1). Both formulations had similar percentages of surface-associated protein (approximately 23\%) and similar in vitro release profiles. Figure 2B shows that VEGF and GDNF released from PLGA NS presented similar release profiles, with an initial burst release of $28 \% \pm 0.5 \%$ of the total loaded protein in the first 24 hours, and a second continuous release rate of $1 \mathrm{ng} / \mathrm{day} /$ $\mathrm{mg}$ persisting until the end of the assay.
Table I Characteristics of the NS

\begin{tabular}{llll}
\hline & Empty-NS & GDNF-NS & VEGF-NS \\
\hline Size $(\mathrm{nm})$ & $267.1 \pm 0.1 \mathrm{I}$ & $221.1 \pm 0.65$ & $235.6 \pm 0.1 \mathrm{I}$ \\
Zeta potential $(\mathrm{mV})$ & $(-) 25.9 \pm 4.44$ & $(-) 22.8 \pm 6.65$ & $(-) 28.9 \pm 6.0 \mathrm{I}$ \\
EE \% & & $74.93 \pm 4.86$ & $44.06 \pm 5.6 \mathrm{I}$ \\
SAP \% & & $22.91 \pm 6.4 \mathrm{I}$ & $23.28 \pm 8.95$ \\
\hline
\end{tabular}

Note: The results are presented as the mean \pm standard deviation.

Abbreviations: EE, encapsulation efficiency; GDNF, glial cell line-derived neurotrophic factor; NS, nanospheres; SAP, surface associated protein; VEGF, vascular endothelial growth factor.

\section{The combination of VEGF and GDNF released from PLGA NS increases cell viability against 6-OHDA}

In this assay, we tested whether VEGF and GDNF released from PLGA NS were able to protect PC-12 cells from 6-OHDA toxicity. As shown in Figure 3A, when the PC-12 cell line was exposed to different 6-OHDA concentrations, the damage to cells was evident under fluorescence microscopy when $0.1 \mathrm{mM}$ and $0.2 \mathrm{mM}$ concentrations were added. These results have been convincingly corroborated by cell viability assay, giving rise to a selection of $0.1 \mathrm{mM}$ 6-OHDA as the ideal dose with which to damage half of the cells (data not shown).

\section{A}
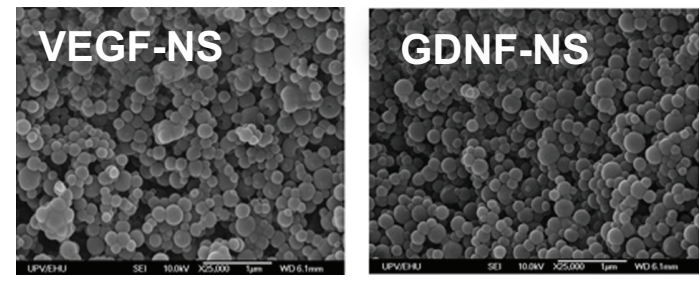

B

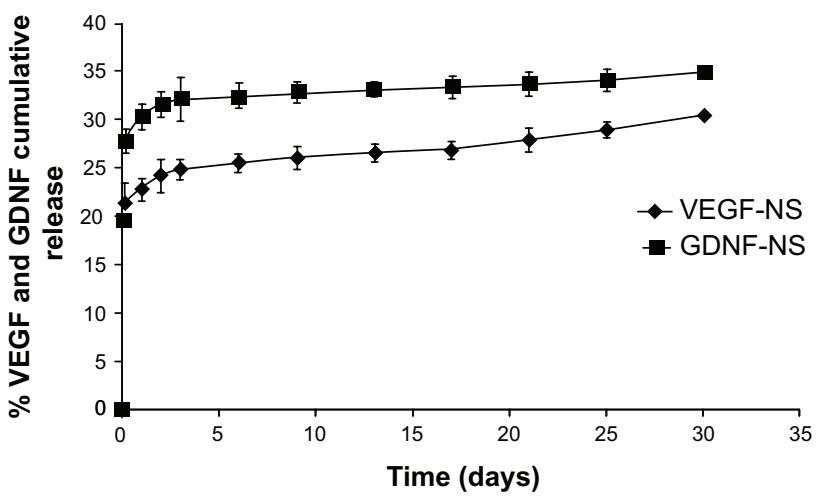

Figure 2 GDNF NS and VEGF NS characterization and in vitro toxicity study. Notes: (A) Scanning electron microscope (SEM) photomicrographs of GDNF NS and VEGF NS. (B) The in vitro VEGF NS and GDNF NS release profiles at $37^{\circ} \mathrm{C}$ in phosphate-buffered saline $(\mathrm{pH}$ 7.4). The results are represented as the mean \pm standard deviation $(n=3)$.

Abbreviations: GDNF, glial cell line-derived neurotrophic factor; NS, nanospheres; VEGF, vascular endothelial growth factor. 
Once the optimal 6-OHDA dose was selected, we found that a combined administration of reduced doses of VEGF and GDNF released from PLGA NS was able to protect dopaminergic cells from 6-OHDA-induced neurotoxicity in vitro. The incubation of PC-12 cells with VEGF $(5 \mathrm{ng} / \mathrm{mL})$ and GDNF $(5 \mathrm{ng} / \mathrm{mL})$ resulted in a $51 \%$ increase in cell viability (negative control: $0.19 \pm 0.03$; VEGF $5 \mathrm{ng} / \mathrm{mL}$ and GDNF $5 \mathrm{ng} / \mathrm{mL}: 0.29 \pm 0.07 ; P<0.001$ with respect to control group, one-way ANOVA [Figure 3B]), achieving a synergistic effect when administered together.

\section{Behavioral study: evaluation of in vivo effects of implanted PLGA NS}

In vivo functionality of nanoencapsulated VEGF and GDNF releasing PLGA NS was assessed by amphetamineinduced rotation test. Only the rats that presented more than three turns/minute after the 6-OHDA lesion were included in the study, and were divided into five experimental groups: 1) vehicle; 2) empty NS; 3) VEGF NS (2.5 $\mu$ g VEGF); 4) GDNF NS (2.5 $\mu \mathrm{g}$ GDNF); and 5) VEGF NS and GDNF NS (1.25 $\mu \mathrm{g}$ VEGF and $1.25 \mu \mathrm{g}$ GDNF). There was no obvious change in the body weight of the rats receiving the different treatments throughout the experiment (data not shown).

In the amphetamine-induced behavioral experiments, no differences were found in the initial number of rotations between groups, as shown in Figure 4A. After a unilateral 6-OHDA lesion, the animals receiving only the vehicle administration did not show significant recovery of the rotational behavior at any time (sham: $10.15 \pm 2.19$ ipsilateral turns/minute at 0 weeks to $11.57 \pm 2.49$ turns/minute at 10 weeks). Rats receiving empty NS, VEGF NS, and
GDNF NS treatments showed a slight reduction in rotations at the end of the study (week 10). GDNF NS implantation, in particular, showed a statistical reduction in the number of rotations when compared with sham group (empty NS: 9.71 \pm 2.92 turns/minute; VEGF NS: $6.37 \pm 2.11$ turns/minute; GDNF NS: $4.43 \pm 2.75$ turns/minute; $P<0.05$ GDNF NS with respect to sham group, Student's $t$-test). In addition, we observed that striatal implantation of the combined VEGF NS and GDNF NS treatment at half the dose of the factors administered individually achieved the best behavioral results and significantly reduced the number of rotations (VEGF NS and GDNF NS: $0.87 \pm 0.53$ turns/minute) when compared to the empty NS and sham groups $(P<0.001$ with respect to empty NS and sham groups, Student's $t$-test [Figure 4A]).

Once the amphetamine test was completed, rats were exposed to the apomorphine-induced test. The data obtained from this test confirmed the promising results obtained previously, showing statistically significant changes between the VEGF NS and GDNF NS group and sham and empty NS treatment groups $(P<0.01$ with respect to empty NS and $P<0.05$ with respect to sham group, Student's $t$-test [Figure 4B]). As can be seen from Figure 4B, the VEGF NS and GDNF NS-treated group presented the smallest number of contralateral rotations, demonstrating the highest level of behavioral recovery.

\section{Histological evaluation of the treatments}

The efficacy of the treatment was also analyzed with immunohistochemical techniques, measuring IOD of TH+ fibers of the striatum and density of dopaminergic neurons in the SN (Figure 5A).
A

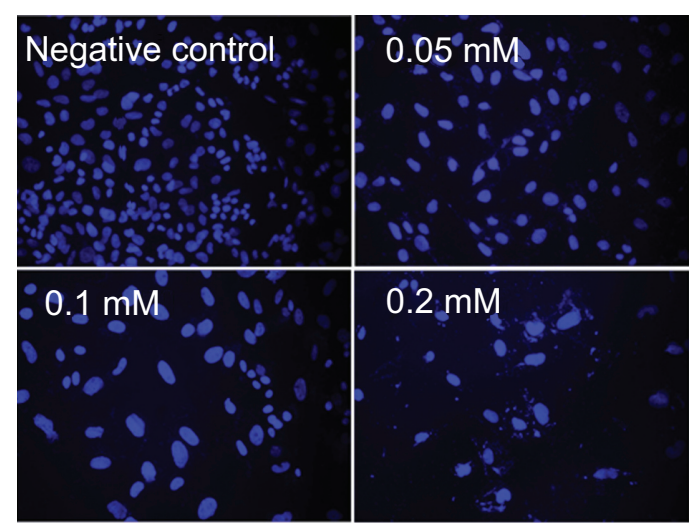

B

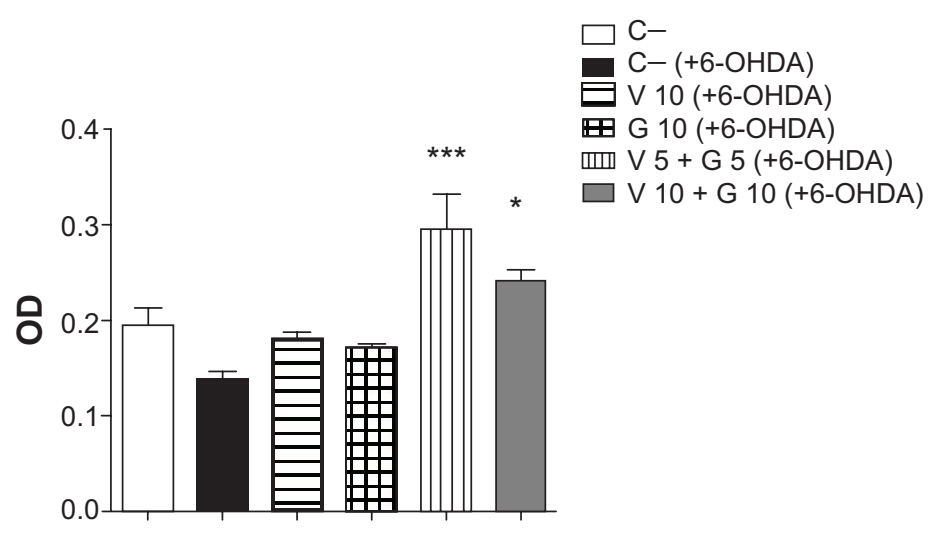

Figure 3 In vitro neurotoxicity assay.

Notes: (A) DAPI immunostaining after different 6-OHDA concentration treatments to establish the neurotoxic cell model. (B) PC-12 cell viability evaluation after 6-OHDA induced toxicity in vitro. ${ }^{*}<<0.05, \mathrm{~V} 10+\mathrm{G} 10$ (+6-OHDA) versus $\mathrm{C}-$, and $\mathrm{C}-(+6-\mathrm{OHDA})$ groups. ***P $<0.00 \mathrm{I}, \mathrm{V} 5+\mathrm{G} 5$ (+6-OHDA) versus $\mathrm{C}-$, and $\mathrm{C}-(+6-\mathrm{OHDA})$ groups. Abbreviations: 6-OHDA, 6-hydroxydopamine; C-, negative control; DAPI, 4',6-diamidino-2-phenylindole; G 5, GDNF 5 ng/mL; G I0, GDNF I0 ng/mL; GDNF, glial cell line-derived neurotrophic factor; OD, optical density; V 5, VEGF $5 \mathrm{ng} / \mathrm{mL}$; V 10, VEGF $10 \mathrm{ng} / \mathrm{mL}$; VEGF, vascular endothelial growth factor. 
A

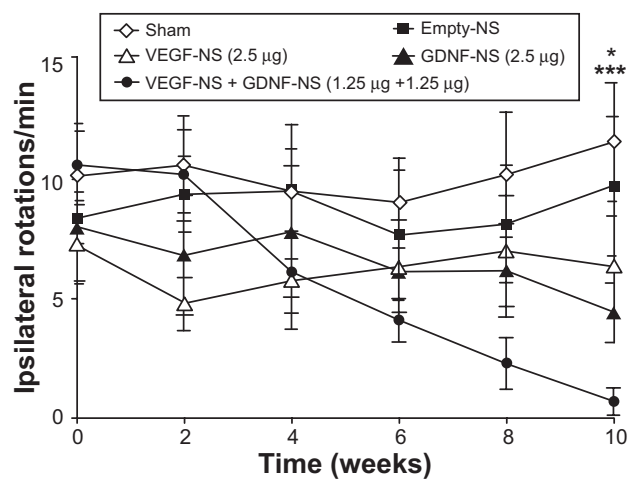

B

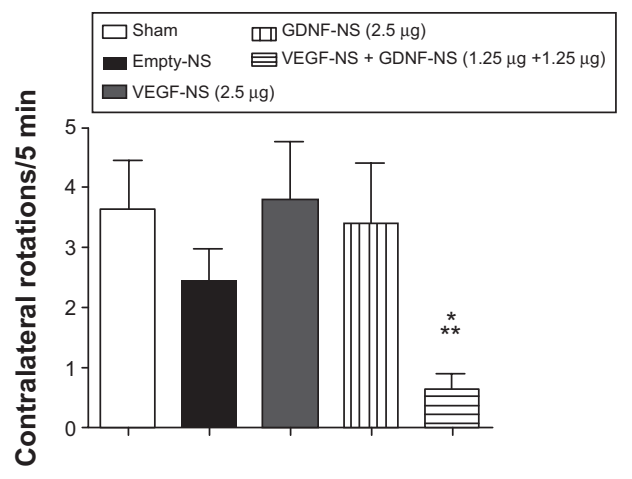

Figure 4 Behavioral study: amphetamine and apomorphine rotational tests.

Notes: $(\mathbf{A})$ The results obtained from the amphetamine rotational behavior test after NS administration. GDNF NS group versus sham group show statistically significant differences $(* P<0.05)$. The VEGF NS and GDNF NS group versus sham and empty NS groups showed statistically significant differences $(* * * P<0.00 \mathrm{I})$. (B) Data obtained from the apomorphine rotational behavior test 14 weeks after NS implantation. The VEGF NS and GDNF NS-treated group exhibited the best behavioral recovery $(* P<0.05$, VEGF NS and GDNF NS versus empty NS; **P<0.01, VEGF NS and GDNF NS versus sham). The data are shown as the mean \pm standard deviation ( $n=6-8)$. Abbreviations: GDNF, glial cell line-derived neurotrophic factor; NS, nanospheres; VEGF, vascular endothelial growth factor.

A

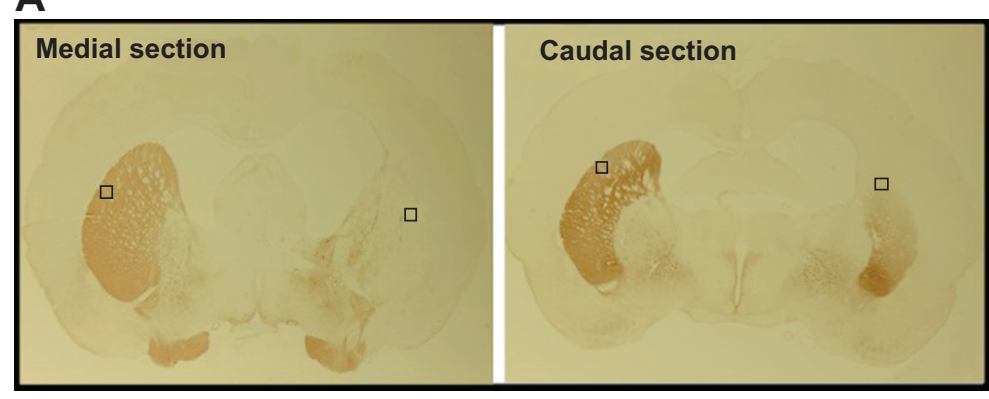

B

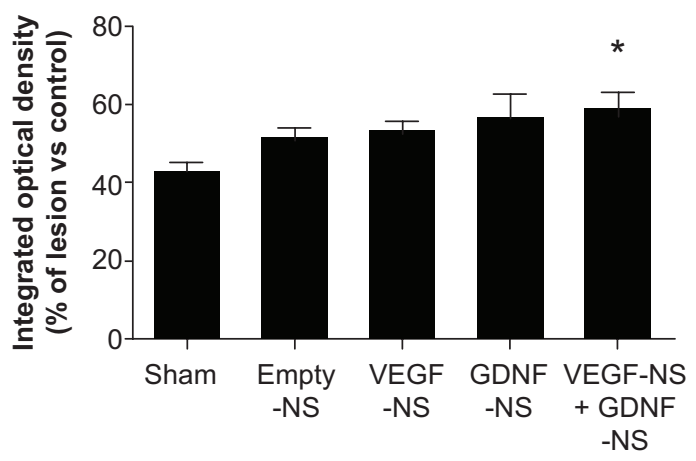

C
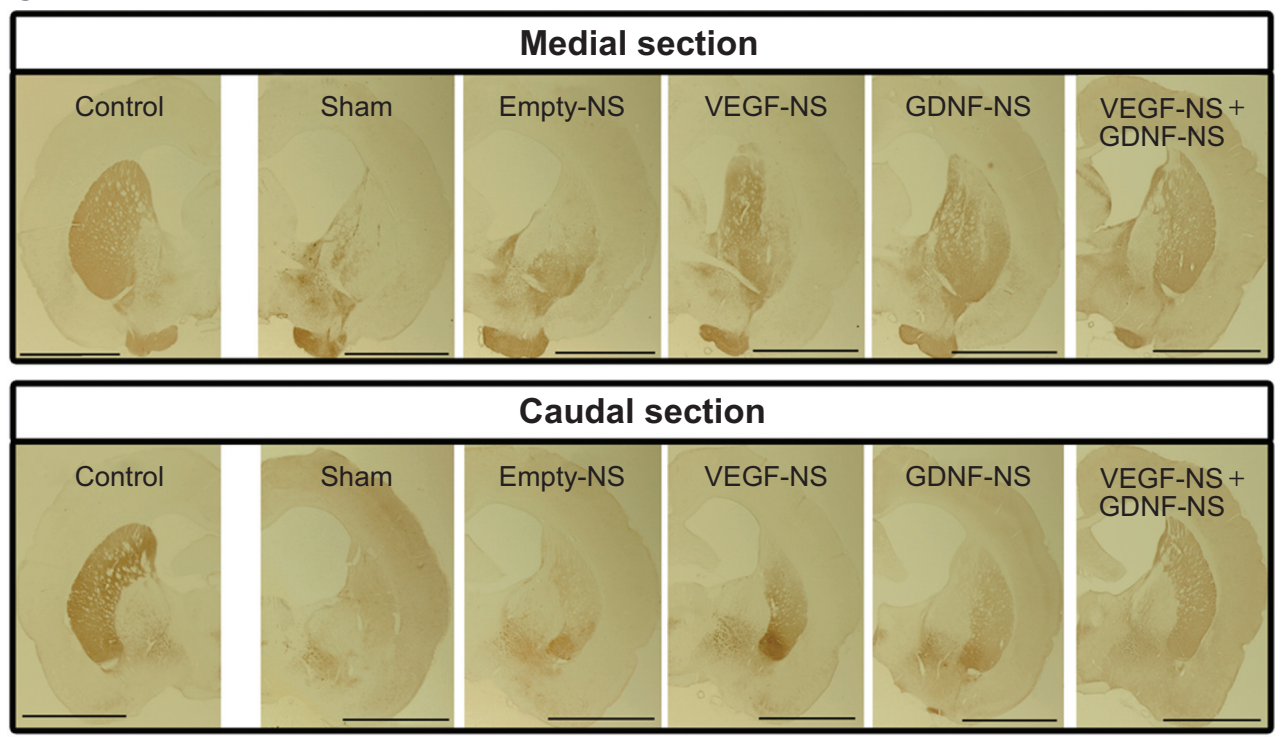

Figure 5 Histological evaluation of the treatments in the striatum.

Notes: (A) The images give an illustrative overview of the medial and caudal sections of the caudoputamen complex for TH immunohistochemistry. Squares placed on lesioned and non-lesioned (control) hemispheres delimit the surfaces where the integrated optical density was measured using a computerized image analysis system. Scale bar $=2 \mathrm{~mm}$. (B) The graphic shows the percentage of integrated optical density of the lesioned hemisphere with respect to the non-lesioned hemisphere (control) for each experimental group. Data are shown as the mean \pm standard error of the mean $(n=6-8)(* P<0.05$ VEGF NS and GDNF NS group versus sham group). (C) Photomicrographs of striata immunostained for TH from a representative intact hemisphere (control) and 6-OHDA lesioned hemispheres from the different experimental groups.

Abbreviations: 6-OHDA, 6-hydroxydopamine; GDNF, glial cell line-derived neurotrophic factor; NS, nanospheres; TH, tyrosine hydroxylase; VEGF, vascular endothelial growth factor. 
From the data shown in Figure 5B, we can see that, 14 weeks after NS implantation, the percentage of $\mathrm{TH}+$ fibers remaining in the ipsilateral hemisphere, in comparison with the non-lesioned half, was greater in the group receiving the combined treatment. The $\mathrm{TH}+$ fiber intensity of the lesioned side expressed as percentage over the control side observed in the striatal area of the VEGF NS and GDNF NS-treated group was $58 \%$, while that of the sham group was $42 \%$. The combined treatment enhanced the restoration of $\mathrm{TH}+$ fibers and neurons to a greater extent than the treatments with only VEGF or GDNF ( $P<0.05$ with respect to the sham group, one-way ANOVA [Figure 5B]). Figure 5C demonstrates enhanced restoration of the lesioned striatum in the VEGF NS and GDNF NS group.

Further evidence of this improvement was found when neuron density of the SN was analyzed. Stereological study showed changes in whole SN neuron density, demonstrating that VEGF NS and GDNF NS increased the density of dopaminergic neurons in a satisfactory way. Moreover, these changes were significantly higher among groups when the "external SN" was considered (Figure 6A and B). The results obtained from the analysis of this specific area showed a considerable increase in neuron density in the VEGF NS and GDNF NS-treated group, the neuronal density being $43 \%$ of that of the contralateral side, compared to only $11 \%$ in the sham cases $(P<0.001$ with respect to sham and empty NS groups, one-way ANOVA [Figure 6C]). This improvement can be clearly observed in the serial pictures of Figure 6D.

\section{Discussion}

Extensive work has been carried out in recent years toward PD treatments aiming at reducing or slowing down the progression of neurodegenerative processes. Concerning new treatments, GFs, and especially neurotrophic factors, offer one of the most compelling opportunities for significantly improving the treatment of this serious neurological disorder, treating both the symptoms of the disease as well as its pathogenesis. ${ }^{27}$

In particular, the efficacy of NTFs such as GDNF, or angiogenic factors like VEGF, in the upregulation of essential neurogenic processes makes them ideal candidates for modifying the evolution of this disorder., ${ }^{4,5,28}$ Previous studies published by our group, as well as research conducted by numerous other laboratories, ${ }^{16,29,30}$ has demonstrated that the administration of VEGF or GDNF improves behavioral deficits as well as decreases neuronal degeneration in different animal models of neurodegenerative disorders. ${ }^{71,20}$

Nevertheless, the essential problems for the application of these hydrophilic molecules are their short half-life and rapid degradation rate in vivo. That is why many studies focus on designing different drug delivery systems to enhance the release of these proteins into the brain tissue, either through invasive or noninvasive methods..$^{31,32}$

Considerable attention has been paid to the improvement of PLGA nanoparticles, making the local delivery of neuroprotective agents into the brain possible. In fact, PLGA NS can preserve the encapsulated unstable therapeutic drug from enzyme degradation, release the drug in a controlled and continuous manner, enhance its biodistribution, and permit drug targeting. In addition, numerous studies have demonstrated that PLGA is well tolerated by the brain tissue without toxic effects, with few adverse effects, and in the absence of immune response. ${ }^{33,34}$

Thus, the aim of this study was to administer VEGFloaded and GDNF-loaded PLGA NS directly into the striatum, and analyze their synergic ability to restore the 6-OHDA-damaged areas, as well as their capability to improve behavioral deficits in a partially lesioned model of PD.

The formulation and characterization of the NS employed in this paper was performed in a similar manner to as reported in our previous work, ${ }^{20}$ generating NS of around $250 \mathrm{~nm}$ particle size and zeta potential around $-30 \mathrm{mV}$, which is considered sufficient to form stable dispersions. In the current study, the in vitro release experiment conducted with VEGF NS and GDNF NS showed a similar biphasic profile with a sustained VEGF and GDNF release for 30 days. Once NS were administered, VEGF and GDNF were released due to diffusion and erosion mechanisms, and, consequently, a constant drug release in therapeutic concentrations in a localized area was obtained, achieving desired effects during the in vivo study.

As soon as the NS were characterized, and with the goal of defining the doses for the in vivo study in 6-OHDA partially lesioned rats, the efficacy of the combined administration of VEGF NS and GDNF NS in the protection of PC-12 cells from degeneration was assessed in vitro. After establishing an in vitro model of PC-12 cell damage using 6-OHDA toxin, we provided new evidence on the protective effect of the mixed administration of VEGF and GDNF released from NS against 6-OHDA neurotoxicity. In this assay, we demonstrated that the combination of VEGF and GDNF increased the cell viability and attenuated 6-OHDA-induced apoptosis, even when half of the dose of the individual factors was administered, showing the synergistic effects of VEGF and GDNF as suggested by Tufro et al. ${ }^{35}$

After the combined administration was successfully proven to be the most effective in PC-12 cell line cultures, we 
A

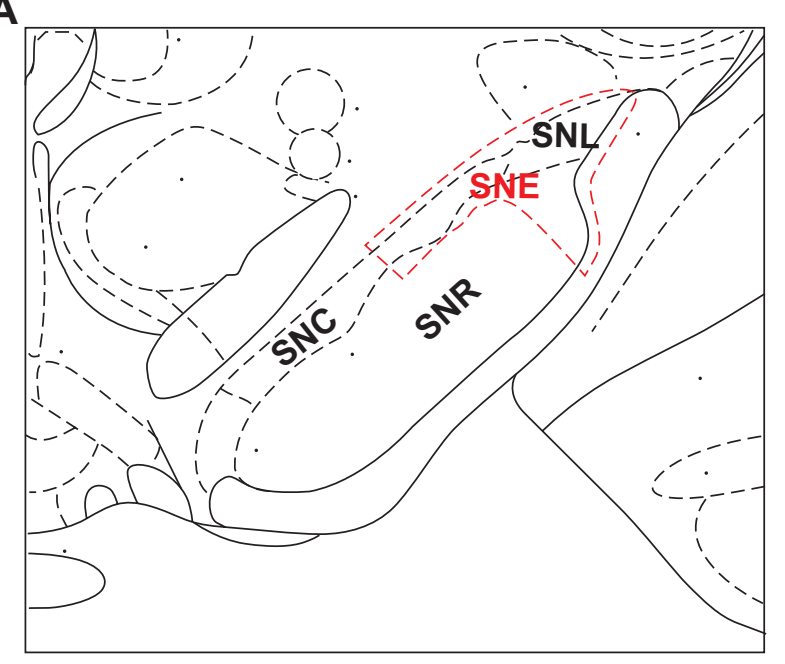

B
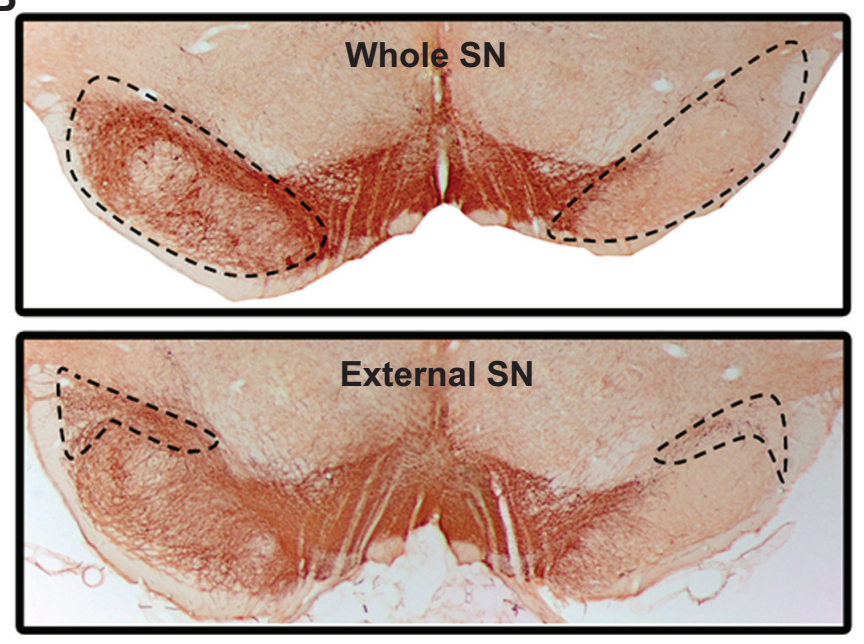

C

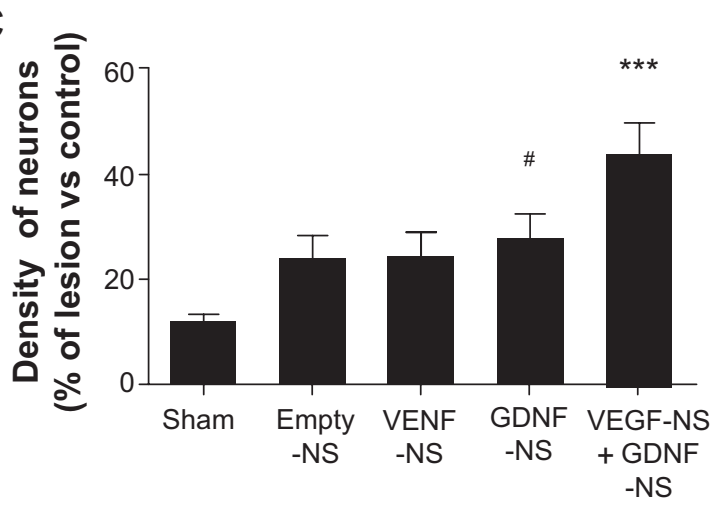

D

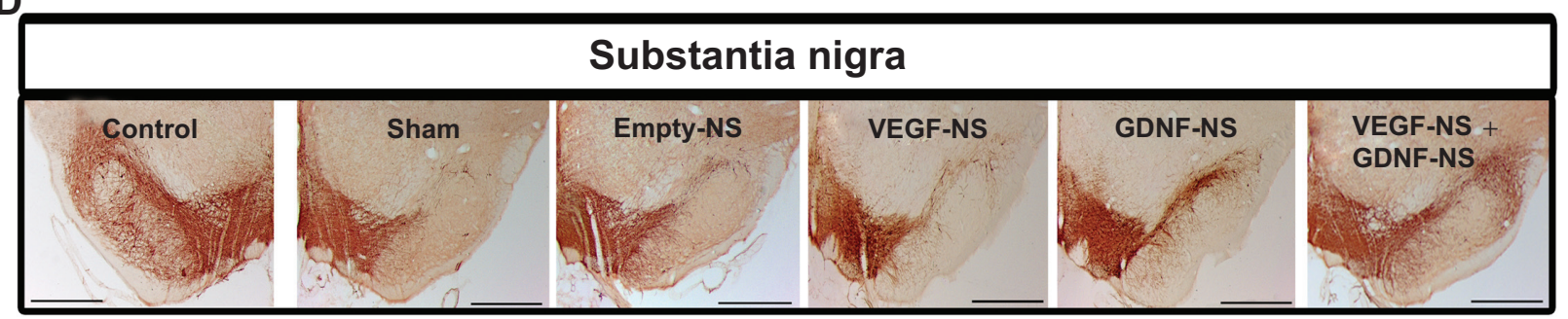

Figure 6 Histological evaluation of the treatments in the SN.

Notes: (A) Schematic illustration of the SN with the "external SN" delimited. This area is topologically related to the lesioned area of striatum and includes SNL, a part of the SNR, and half of the SNC. (B) Picture of whole SN and delimited "external SN". Scale bar =I mm. (C) Density of dopaminergic neurons in "external SN". The results are expressed as a percentage of lesioned hemisphere compared to the non-lesioned one (control). Data are shown as the mean \pm standard error of the mean ( $\mathrm{n}=6-8$ ) ( $\#<0.05$ GDNF NS group versus sham group; $* * * P<0.00$ I VEGF NS and GDNF NS group versus sham and empty NS groups). (D) Photomicrographs of SN immunostained for tyrosine hydroxylase from a representative intact hemisphere (control) and 6-OHDA lesioned hemispheres from the different experimental groups. Scale bar =I mm. Abbreviations: 6-OHDA, 6-hydroxydopamine; GDNF, glial cell line-derived neurotrophic factor; NS, nanospheres; SN, substantia nigra; SNC, SN pars compacta; SNL, SN lateral; SNR, SN pars reticulata; SNE, SN externa; VEGF, vascular endothelial growth factor.

investigated the in vivo potential of the combined administration at lower doses of VEGF NS and GDNF NS for inducing the restoration of the damage brain areas. The data of this new in vivo study showed improvements on the results obtained in our previous work, carried out in a totally lesioned rat model, using microspheres to administer the factors. ${ }^{\text {? }}$

In the present study, we selected a partially lesioned rat model, with the aim of proving the efficacy of our therapy at an incipient stage of PD and with the possibility of comparing with other similar studies published by Garbayo et al and Jollivet et al. ${ }^{29,30}$ During the 10 weeks of treatment, we corroborated the behavioral benefits generated by VEGF NS and GDNF NS. In the amphetamine-induced rotation test, the results obtained from GDNF NS treatment were consistent with those of other studies and corroborated the beneficial effects of GDNF in the behavioral recovery. Furthermore, 
VEGF NS and GDNF NS combined treatment presented a lower number of ipsilateral rotations, demonstrating higher recovery levels when compared with other treatment groups in the lesioned animals. This result was supported by the experimental data obtained from the apomorphine-induced rotation test. Therefore, the positive behavioral results obtained in the combined treatment group suggest that a sufficient recovery in the lesioned brain tissue could have occurred.

Because the injection of 6-OHDA is directly related to the loss of dopaminergic neurons, and, consequently, is associated with disturbed behavioral function, we investigated the number of $\mathrm{TH}+$ fibers in the striatum of the lesioned rats. The immunohistochemical analysis correlated with the result obtained in the behavioral study, showing increased fiber density in the striatum in the combined treatment group. A particularly interesting finding was the statistically significant changes found in "external SN" dopaminergic neurons after GDNF NS and VEGF NS and GDNF NS treatments. It is well documented that neurons located on the most "external SN" are topologically related to the dorsolateral caudoputamen complex, corresponding to the most lesioned area in this partially lesioned PD rat model. Thus, the restorative changes we observed in SN may be due to the activity of GFs in the striatum. ${ }^{21,26}$ These findings support the neurorestorative role of nanoencapsulated VEGF and GDNF on the dopaminergic system, where functional improvement was accompanied with a morphological restoration in a partial-lesion model of PD. The synergistic effects of VEGF NS and GDNF NS allow for a reduction of the dose by half, thus reducing possible side effects, and is a promising neuroregenerative/neuroreparative approach for the treatment of PD.

\section{Conclusion}

The data obtained from behavioral studies and immunohistochemical analysis of the striatum and SN confirm that the combination of VEGF NS and GDNF NS at half the dose of their individual administration can be a suitable and promising treatment for neuronal regeneration and protection in a partially lesioned rat model of PD. In addition, the measurements focused on the "external SN" achieve more specific and significant results. Therefore, the administration of VEGF NS and GDNF NS is an interesting potential strategy for the treatment of PD.

\section{Acknowledgments}

The "Ministerio de Ciencia e Innovación" (SAF2010-20375), the University of the Basque Country (UPV/EHU) (UFI 11/32), the Basque Government (Saiotek SA-2010/00028), and FEDER funds partially supported this project. The authors are grateful for the cooperation of SGIker (UPV/ EHU, MICINN, GV/EJ, ESF). E Herrán appreciates the Basque Government fellowship subvention, and C Requejo and A Aristieta thank UPV/EHU for a fellowship subvention. We thank Dr A Krzyzanowska for the careful revision of the manuscript.

\section{Disclosure}

The authors report no conflicts of interest in this work.

\section{References}

1. Linazasoro G. A global view of Parkinson's disease pathogenesis: implications for natural history and neuroprotection. Parkinsonism Relat Disord. 2009;15(6):401-405.

2. Obeso JA, Rodriguez-Oroz MC, Goetz CG, et al. Missing pieces in the Parkinson's disease puzzle. Nat Med. 2010;16(6):653-661.

3. Deierborg T, Soulet D, Roybon L, Hall V, Brundin P. Emerging restorative treatments for Parkinson's disease. Prog Neurobiol. 2008;85(4): 407-432.

4. Sullivan AM, Toulouse A. Neurotrophic factors for the treatment of Parkinson's disease. Cytokine Growth Factor Rev. 2011;22(3):157-165.

5. Ruozi B, Belletti D, Bondioli L, et al. Neurotrophic factors and neurodegenerative diseases: a delivery issue. Int Rev Neurobiol. 2012;102: 207-247.

6. Siegel GJ, Chauhan NB. Neurotrophic factors in Alzheimer's and Parkinson's disease brain. Brain Res Brain Res Rev. 2000;33(2-3): 199-227.

7. Herrán E, Ruiz-Ortega JÁ, Aristieta A, et al. In vivo administration of VEGF- and GDNF-releasing biodegradable polymeric microspheres in a severe lesion model of Parkinson's disease. Eur J Pharm Biopharm. 2013;85(3 Pt B):1183-1190.

8. Zeng X, Chen J, Deng X, et al. An in vitro model of human dopaminergic neurons derived from embryonic stem cells: MPP+ toxicity and GDNF neuroprotection. Neuropsychopharmacology. 2006; 31(12):2708-2715.

9. Theofilopoulos S, Goggi J, Riaz SS, Jauniaux E, Stern GM, Bradford HF. Parallel induction of the formation of dopamine and its metabolites with induction of tyrosine hydroxylase expression in foetal rat and human cerebral cortical cells by brain-derived neurotrophic factor and glial-cell derived neurotrophic factor. Brain Res Dev Brain Res. 2001; 127(2):111-122.

10. Eggert K, Schlegel J, Oertel W, Würz C, Krieg J, Vedder H. Glial cell line-derived neurotrophic factor protects dopaminergic neurons from 6-hydroxydopamine toxicity in vitro. Neurosci Lett. 1999;269(3): $178-182$.

11. Grandoso L, Ponce S, Manuel I, et al. Long-term survival of encapsulated GDNF secreting cells implanted within the striatum of parkinsonized rats. Int J Pharm. 2007;343(1-2):69-78.

12. Lindvall O, Wahlberg LU. Encapsulated cell biodelivery of GDNF: a novel clinical strategy for neuroprotection and neuroregeneration in Parkinson's disease? Exp Neurol. 2008;209(1):82-88.

13. Nutt JG, Burchiel KJ, Comella CL, et al; ICV GDNF Study Group. Implanted intracerebroventricular. Glial cell line-derived neurotrophic factor. Randomized, double-blind trial of glial cell linederived neurotrophic factor (GDNF) in PD. Neurology. 2003;60(1): 69-73.

14. Lang AE, Gill S, Patel NK, et al. Randomized controlled trial of intraputamenal glial cell line-derived neurotrophic factor infusion in Parkinson disease. Ann Neurol. 2006;59(3):459-466.

15. Slevin JT, Gash DM, Smith CD, et al. Unilateral intraputamenal glial cell line-derived neurotrophic factor in patients with Parkinson disease: response to 1 year of treatment and 1 year of withdrawal. J Neurosurg. 2007;106(4):614-620. 
16. Yasuhara T, Shingo T, Kobayashi K, et al. Neuroprotective effects of vascular endothelial growth factor (VEGF) upon dopaminergic neurons in a rat model of Parkinson's disease. Eur J Neurosci. 2004;19: 1494-1504.

17. Pitzer MR, Sortwell CE, Daley BF, et al. Angiogenic and neurotrophic effects of vascular endothelial growth factor (VEGF165): studies of grafted and cultured embryonic ventral mesencephalic cells. Exp Neurol. 2003;182(2):435-445.

18. Talmadge JE. The pharmaceutics and delivery of therapeutic polypeptides and proteins. Adv Drug Deliv Rev. 1993;10(2-3):247-299.

19. Chen Y, Liu L. Modern methods for delivery of drugs across the bloodbrain barrier. Adv Drug Deliv Rev. 2012;64(7):640-665.

20. Herrán E, Pérez-González R, Igartua M, Pedraz JL, Carro E, Hernández RM. VEGF-releasing biodegradable nanospheres administered by craniotomy: a novel therapeutic approach in the APP/Ps1 mouse model of Alzheimer's disease. J Control Release. 2013;170(1): 111-119.

21. Deumens R, Blokland A, Prickaerts J. Modeling Parkinson's disease in rats: an evaluation of 6-OHDA lesions of the nigrostriatal pathway. Exp Neurol. 2002;175(2):303-317.

22. Björklund A, Rosenblad C, Winkler C, Kirik D. Studies on neuroprotective and regenerative effects of GDNF in a partial lesion model of Parkinson's disease. Neurobiol Dis. 1997;4(3-4):186-200.

23. Morera-Herreras T, Ruiz-Ortega JA, Linazasoro G, Ugedo L. Nigrostriatal denervation changes the effect of cannabinoids on subthalamic neuronal activity in rats. Psychopharmacology (Berl). 2011;214(2): 379-389.

24. Miguelez C, Aristieta A, Cenci MA, Ugedo L. The locus coeruleus is directly implicated in L-DOPA-induced dyskinesia in parkinsonian rats: an electrophysiological and behavioural study. PLoS One. 2011;6(9):e24679.

25. Paxinos G, Watson C. The Rat Brain in Steretaxic Coordinates. Academic Press, San Diego, CA, USA. 1997.

26. Rosenblad C, Kirik D, Björklund A. Sequential administration of GDNF into the substantia nigra and striatum promotes dopamine neuron survival and axonal sprouting but not striatal reinnervation or functional recovery in the partial 6-OHDA lesion model. Exp Neurol. 2000;161(2):503-516.
27. Bartus RT, Baumann TL, Brown L, Kruegel BR, Ostrove JM, Herzog CD Advancing neurotrophic factors as treatments for age-related neurodegenerative diseases: developing and demonstrating "clinical proofof-concept" for AAV-neurturin (CERE-120) in Parkinson's disease. Neurobiol Aging. 2013;34(1):35-61.

28. Youdim MB, Kupershmidt L, Amit T, Weinreb O. Promises of novel multi-target neuroprotective and neurorestorative drugs for Parkinson's disease. Parkinsonism Relat Disord. 2014;20 Supp1 1:S132-S136.

29. Garbayo E, Montero-Menei CN, Ansorena E, Lanciego JL, Aymerich MS, Blanco-Prieto MJ. Effective GDNF brain delivery using microspheres - a promising strategy for Parkinson's disease. J Control Release. 2009;135(2):119-126.

30. Jollivet C, Aubert-Pouessel A, Clavreul A, et al. Striatal implantation of GDNF releasing biodegradable microspheres promotes recovery of motor function in a partial model of Parkinson's disease. Biomaterials. 2004;25(5):933-942.

31. Garbayo E, Ansorena E, Blanco-Prieto MJ. Drug development in Parkinson's disease: from emerging molecules to innovative drug delivery systems. Maturitas. 2013;76(3):272-278.

32. Stockwell J, Abdi N, Lu X, Maheshwari O, Taghibiglou C. Novel central nervous system drug delivery systems. Chem Biol Drug Des. 2014;83(5):507-520.

33. Pridgen EM, Langer R, Farokhzad OC. Biodegradable, polymeric nanoparticle delivery systems for cancer therapy. Nanomedicine (Lond). 2007;2(5):669-680.

34. Yamamoto H, Tahara K, Kawashima Y. Nanomedical system for nucleic acid drugs created with the biodegradable nanoparticle platform. J Microencapsul. 2012;29(1):54-62.

35. Tufro A, Teichman J, Banu N, Villegas G. Crosstalk between VEGF-A/ VEGFR2 and GDNF/RET signaling pathways. Biochem Biophys Res Commun. 2007;358(2):410-416.
International Journal of Nanomedicine

\section{Publish your work in this journal}

The International Journal of Nanomedicine is an international, peerreviewed journal focusing on the application of nanotechnology in diagnostics, therapeutics, and drug delivery systems throughout the biomedical field. This journal is indexed on PubMed Central, MedLine, CAS, SciSearch $\AA$, Current Contents $₫ /$ Clinical Medicine,

\section{Dovepress}

Journal Citation Reports/Science Edition, EMBase, Scopus and the Elsevier Bibliographic databases. The manuscript management system is completely online and includes a very quick and fair peer-review system, which is all easy to use. Visit http://www.dovepress.com/ testimonials.php to read real quotes from published authors. 